\title{
DETC2001/VIB-21615
}

\section{EFFICIENT DYNAMIC SIMULATION OF FLEXIBLE LINK MANIPULATORS WITH PID CONTROL}

\author{
Ronald G.K.M. Aarts \\ Department of Mechanical Engineering \\ University of Twente \\ P.O. Box 217, 7500 AE Enschede \\ The Netherlands \\ Email: R.G.K.M.Aarts@wb.utwente.nl \\ WWW: http://www.wa.wb.utwente.nl/
}

\author{
J.B. Jonker \\ Department of Mechanical Engineering \\ University of Twente \\ P.O. Box 217, 7500 AE Enschede \\ The Netherlands \\ Email: J.B.Jonker@wb.utwente.nl \\ WWW: http://www.wa.wb.utwente.nl/
}

\begin{abstract}
For accurate simulations of the dynamic behavior of flexible manipulators the combination of a perturbation method and modal analysis is proposed. First, the vibrational motion is modeled as a first-order perturbation of a nominal rigid link motion. The vibrational motion is then described by a set of linear timevarying equations. Next, the number of degrees of freedom is reduced by applying a modal reduction technique. The proportional part of the control system is explicitly included in the modal analysis. The applicability of the method is demonstrated by simulating the controlled trajectory motion of a spatial flexible three-degree of freedom manipulator with PID control.
\end{abstract}

\section{INTRODUCTION}

In this paper a perturbation method is proposed for analyzing the dynamic behavior of flexible manipulators, including the effects of the manipulators control system. It involves a non-linear finite element formulation [1] in which links and joints are considered as specific elements. The governing equations of motion are derived in two sets of coordinates. The first set are the generalized coordinates of the manipulator with rigid links which are used to express control forces and torques. The second set are deformation coordinates that characterize flexible deformations of the links. The non-linear differential equations are solved using a two-step perturbation approach. In this approach the vi- brational motion of the manipulator is modeled as a first-order perturbation of the nominal rigid link motion. In the first step the nominal rigid link motion is described by the rigidified manipulator model, that is a non-linear model in which all flexible deformation coordinates are prescribed zero. In a dynamic analysis the equations of motion are symbolically linearized and evaluated numerically in a number of points of the nominal trajectory. During the second step the vibrational motion is described by a set of linear time-varying equations using the linearized description.

In order to reduce the dimension of the linearized system, a modal reduction technique is proposed. The mode shape functions are determined by taking into account the proportional feedback gains associated with the rigid link motion. Furthermore, the time-varying nature of the mode shape functions is taken into account. Then modal integration can be applied using only a small number of low-frequency modes. The modeacceleration concept is used to account for the pseudo-static contribution of the high-frequency modes.

A spatial flexible three-degree of freedom manipulator (Fig. 1) is analyzed to illustrate the solution method. Results from a non-linear simulation are compared with the perturbation method with and without modal reduction. In earlier papers we discussed the applicability of the non-linear simulations [2,3] and the perturbation method [4]. In this paper we will focus on the modal analysis. 


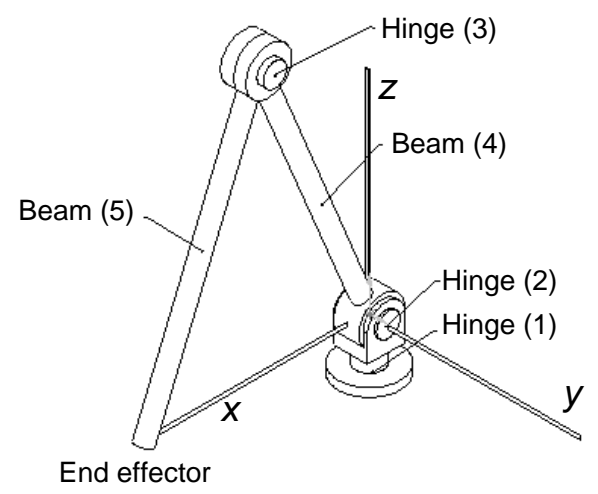

Figure 1. Spatial two link manipulator.

\section{NON-LINEAR EQUATIONS OF MOTION}

A non-linear finite element method is used in which a manipulator mechanism is modeled as an assembly of finite elements interconnected by joint elements such as hinge elements and beam elements. The location of each element is described relative to a fixed inertial coordinate system by a set of nodal coordinates $x_{i}$. With respect to some reference configuration of the element, the instantaneous values of the nodal coordinates represent a fixed number of deformation modes for the element. The deformation modes are specified by a set of deformation parameters $\left(e_{i}, \varepsilon_{i}\right)$. The deformation parameters $e_{i}$ are associated with large relative displacements and rotations between the element nodes, while the $\varepsilon_{i}$ describe small elastic deformations of the elements. The deformation parameters can be expressed as analytic functions of the nodal coordinates. For a detailed description of these so-called deformation functions the reader is referred to $[1,5]$.

The motion of manipulator mechanisms is described by relative degrees of freedom, which can be either actuator joint coordinates, denoted $\underline{e}^{m}$, as well as flexible deformation coordinates denoted by $\underline{\varepsilon}^{m}$. The superscript $m$ is used to denote the degrees of freedom. By means of the geometric transfer function $\mathcal{E}$ the nodal coordinates are expressed as

$$
\underline{x}=\underline{\mathcal{E}}\left(\underline{e}^{m}, \underline{\varepsilon}^{m}\right)
$$

By differentiating the transfer function, we obtain

$$
\underline{\dot{x}}=\mathrm{D} \underline{\mathcal{F}} \cdot\left(\underline{\dot{e}}^{m}, \underline{\dot{\varepsilon}}^{m}\right),
$$

and for the second derivative

$$
\underline{\ddot{x}}=\left(\mathrm{D}^{2} \mathcal{E} \cdot\left(\underline{\dot{e}}^{m}, \underline{\dot{\varepsilon}}^{m}\right)\right) \cdot\left(\underline{\dot{e}}^{m}, \underline{\dot{\varepsilon}}^{m}\right)+\mathrm{D} \underline{\mathcal{E}} \cdot\left(\underline{\ddot{e}}^{m}, \underline{\ddot{\varepsilon}}^{m}\right),
$$

where the differentiation operator D is used to represent partial differentiation with respect to the vector of the degrees of free$\operatorname{dom}\left(\underline{e}^{m}, \underline{\varepsilon}^{m}\right)$.

The inertia properties of the concentrated and distributed mass of the elements are described with the aid of lumped and consistent mass matrices [5-7]. Let $M(\underline{x})$ be the global mass matrix, obtained by assembling the lumped and consistent element mass matrices, and let $f(\underline{x}, \underline{\dot{x}}, t)$ be the vector of external nodal forces, including gravitational forces and the velocity dependent inertia forces. The loading state of each element is described by a vector of stress resultants. The vectors are assembled in the global vectors $\underline{\sigma}^{e}$ and $\underline{\sigma}^{\varepsilon}$. Application of the principle of virtual power yields the reduced equations of motion

$$
\begin{aligned}
& {\left[\begin{array}{ll}
\bar{M}^{e e} & \bar{M}^{e \varepsilon} \\
\bar{M}^{\varepsilon e} & \bar{M}^{\varepsilon \varepsilon}
\end{array}\right]\left[\begin{array}{l}
\ddot{\ddot{e}}^{m} \\
\underline{\ddot{\varepsilon}}^{m}
\end{array}\right]} \\
& +\left[\begin{array}{c}
\mathrm{D}_{e^{m}} \mathcal{\mathcal { F }}^{T} \\
\mathrm{D}_{\varepsilon^{m}} \mathcal{E}^{T}
\end{array}\right]\left[M\left(\mathrm{D}^{2} \mathcal{F} \cdot\left(\underline{\dot{e}}^{m}, \underline{\dot{\varepsilon}}^{m}\right)\right) \cdot\left(\underline{\dot{e}}^{m}, \underline{\dot{\varepsilon}}^{m}\right)-f\right]=-\left[\begin{array}{l}
\underline{\sigma}^{e m} \\
\underline{\underline{\sigma}}^{\varepsilon m}
\end{array}\right] .
\end{aligned}
$$

The $\underline{e}^{m}$ and $\underline{\varepsilon}^{m}$ parts of the reduced mass matrix $\bar{M}=\mathrm{D} \underline{\mathcal{F}}^{T} M \mathrm{D} \mathcal{F}$ are explicitly indicated. The stress resultant vector of flexible elements is characterized by Hooke's law defined by $\underline{\sigma}^{\varepsilon}=K^{\varepsilon \varepsilon} \underline{\varepsilon}$, where $K^{\varepsilon \varepsilon}$ is a symmetric matrix containing the elastic constants. The driving forces and torques, represented by the vector $\underline{\sigma}^{e m}$, are applied only at the actuator joints. If actuator dynamics are not considered then there is a simple linear relation between the vector of control inputs $\underline{u}$ and the vector $\underline{\sigma}^{\text {em }}$

$$
\underline{\sigma}^{e m}=-\underline{u}
$$

By defining a vector of generalized coordinates

$$
\underline{q}=\left[\begin{array}{l}
\underline{e}^{m} \\
\underline{\varepsilon}^{m}
\end{array}\right]
$$

and using Eq. (5) and Hooke's law, the equations of motion (4) can be written in a more compact form

$$
\bar{M}(\underline{q}) \ddot{\underline{q}}+\mathrm{D} \underline{\mathcal{E}}^{T}\left[M\left(\mathrm{D}^{2} \underline{\mathcal{E}} \cdot \dot{\underline{q}}\right) \cdot \dot{\underline{q}}-f\right]+K \underline{q}=B \underline{u},
$$

where the structural stiffness matrix $K$ and the input matrix $B$ are

$$
K=\left[\begin{array}{cc}
0 & 0 \\
0 & K^{\varepsilon \varepsilon}
\end{array}\right], \quad B=\left[\begin{array}{l}
I \\
0
\end{array}\right]
$$

\section{PERTURBATION METHOD}

Given the non-linear equations of motion in Eq. (7), consider now small perturbations around the nominal trajectory 
$\left(\underline{q}_{0}, \dot{q}_{0}, \ddot{q}_{0}\right)$ such that the actual variables are of the form

$$
\begin{aligned}
& \underline{q}=\underline{q}_{0}+\delta \underline{q}, \\
& \dot{q}=\dot{q}_{0}+\delta \dot{q}, \\
& \ddot{q}=\ddot{q}_{0}+\delta \ddot{q},
\end{aligned}
$$

where the prefix $\delta$ denotes a perturbation. Expanding Eq. (1) in its Taylor series expansion and disregarding second and higher order terms results in the linear approximation

$$
\delta \underline{x}=\mathrm{D} \underline{\mathcal{F}} \delta \underline{q} .
$$

Linearization of the reduced equations of motion (7) around the nominal trajectory $\left(\underline{q}_{0}, \dot{q}_{0}, \ddot{q}_{0}\right)$ results in

$$
\bar{M}_{0} \delta \ddot{q}+C_{0} \delta \dot{q}+\left(K_{0}+G_{0}+N_{0}\right) \delta \underline{q}=B \delta \underline{u},
$$

where $\bar{M}_{0}$ is the system mass matrix as in (7), $C_{0}$ is the velocity sensitivity matrix, and $K_{0}$ denotes the structural stiffness matrix as in (8), $G_{0}$ and $N_{0}$ are the geometric stiffening matrix and the dynamic stiffening matrix, respectively. $\bar{M}_{0}, K_{0}$ and $G_{0}$ are symmetric matrices, but $C_{0}$ and $N_{0}$ need not. These matrices are calculated using an analytical linearization method $[7,8]$. The matrix coefficients depend on the nominal position, velocity and acceleration of the manipulator and consequently they are timevarying.

According to the perturbation method the equations of motion are separated into a set of non-linear equations for the nominal rigid link motion and a set of linearized equations describing small vibrational motions and deviations from the rigid link motion. The nominal rigid link motion is described by the rigidified model, that is a model in which all vibrational deformations are prescribed zero. The rigidified model is calculated by setting $\underline{\ddot{\varepsilon}}^{m}=\underline{\dot{\varepsilon}}^{m}=\underline{\varepsilon}^{m}=\underline{0}$ in Eq. (4), yielding

$$
\begin{aligned}
& \bar{M}_{0}^{e e} \underline{\underline{e}}_{0}^{m}+\mathrm{D}_{e^{m}} \underline{\mathcal{F}}_{0}^{T} \\
& {\left[M_{0}\left(\mathrm{D}^{2} \underline{\mathcal{F}}_{0} \cdot\left(\underline{\dot{e}}_{0}^{m}, \underline{0}\right)\right) \cdot\left(\underline{\dot{e}}_{0}^{m}, \underline{0}\right)-f\right]=\underline{u}_{0},} \\
& \bar{M}_{0}^{\varepsilon e} \underline{\ddot{e}}_{0}^{m}+\mathrm{D}_{\varepsilon^{m}} \underline{\mathcal{F}}_{0}^{T} \\
& \quad\left[M_{0}\left(\mathrm{D}^{2} \underline{\mathcal{F}}_{0} \cdot\left(\underline{\dot{e}}_{0}^{m}, \underline{0}\right)\right) \cdot\left(\underline{\dot{e}}_{0}^{m}, \underline{0}\right)-f\right]=-\underline{\sigma}_{0}^{\varepsilon m},
\end{aligned}
$$

where $\underline{u}_{0}$ represents the vector of nominal input forces and torques necessary to move the rigid link manipulator along the nominal (desired) trajectory. The vector $\sigma_{0}^{\varepsilon m}$ describes the generalized stress resultants (Lagrange multipliers) of the rigidified links. These forces are balanced by the internal excitation forces of the vibrational motion of the links. They are therefore added to the righthand side of the linearized equations of motion with reversed sign. The vibrational motion is then described by a set of inhomogeneous linear time-varying equations of the form

$$
\begin{aligned}
\bar{M}_{0}\left[\begin{array}{c}
\delta \underline{\ddot{e}}^{m} \\
\underline{\ddot{\varepsilon}}^{m}
\end{array}\right]+C_{0}\left[\begin{array}{c}
\delta \underline{\dot{e}}^{m} \\
\underline{\dot{\varepsilon}}^{m}
\end{array}\right] \\
\quad+\left[K_{0}+G_{0}+N_{0}\right]\left[\begin{array}{l}
\delta \underline{e}^{m} \\
\underline{\varepsilon}^{m}
\end{array}\right]=\left[\begin{array}{c}
\delta \underline{u} \\
\underline{\sigma}_{0}^{\varepsilon m}
\end{array}\right],
\end{aligned}
$$

where $\underline{\varepsilon}^{m}=\delta \underline{\varepsilon}^{m}$ as $\underline{\varepsilon}_{0}^{m}=0$ and the vector $\delta \underline{u}$ is the control input vector synthesized at the stage of perturbed dynamics. For the modal analysis in the next section we explicitly consider the "stiffness" of the applied control system. To account for this contribution it is assumed that the control input vector $\delta \underline{u}$ can be written as

$$
\delta \underline{u}=-K_{p} \delta \underline{e}^{m}+\delta \underline{u}_{\mathrm{d}},
$$

where the matrix $K_{p}$ represents the proportional action of the controller and the remaining dynamic action is described by $\delta u_{\mathrm{d}}$. Substituting Eqs. (6) and (14) into Eq. (13) leads to a shorthand notation

$$
\bar{M}_{0} \delta \ddot{q}+C_{0} \delta \dot{q}+\bar{K}_{0} \delta \underline{q}=\underline{\sigma}_{0},
$$

where the righthand side vector is

$$
\underline{\sigma}_{0}=\left[\begin{array}{l}
\delta \underline{u}_{\mathrm{d}} \\
\underline{\sigma}_{0}^{\varepsilon m}
\end{array}\right]
$$

The combined stiffness matrix $\bar{K}_{0}$ is defined as

$$
\bar{K}_{0}=K_{0}+G_{0}+N_{0}+\left[\begin{array}{rr}
K_{p} & 0 \\
0 & 0
\end{array}\right]
$$

to include the proportional control matrix $K_{p}$.

\section{ADAPTIVE MODAL INTEGRATION METHOD}

In the linearized equation (15) both low and high-frequency modes are taken into account. Usually, the largest amplitudes are observed at low frequencies whereas the presence of high frequencies significantly slows down the simulations. In linear structural dynamics, modal analysis techniques are used to improve the computational efficiency. With some modifications these modal techniques can also be applied to solve Eq. (15).

Analogously to e.g. Craig [9] the key step in the modesuperposition method is the introduction of a coordinate transformation

$$
\delta \underline{q}=\Phi \underline{\underline{\eta}}
$$


where the $n$ elements of the vector $\underline{\eta}$ are the so-called principal coordinates $\eta_{i}$. The columns of the modal matrix $\Phi$ are the natural modes $\Phi_{i}$. These modes and the natural frequencies $\omega_{i}$ are then found by solving the eigenvalue problem with the mass and stiffness matrices $\bar{M}_{0}$ and $\bar{K}_{0}$. Unfortunately, the dynamic stiffening term $N_{0}$ in the stiffness matrix is in general not symmetric. Hence the system described by $\bar{M}_{0}$ and $\bar{K}_{0}$ is circulatory and the solution of the eigenvalue problem may lead to natural frequencies and modes with imaginary parts [10]. To avoid these solutions we consider the eigenvalue problem

$$
\left(\bar{K}_{0}^{S}-\omega_{i}^{2} \bar{M}_{0}\right) \underline{\phi}_{i}=0 \quad(i=1,2, \ldots, n),
$$

in which a symmetric stiffness matrix is used

$$
\bar{K}_{0}^{S}=\frac{1}{2}\left(\bar{K}_{0}+\bar{K}_{0}^{T}\right)
$$

The mass and stiffness matrices are time-varying, so the natural frequencies and the modal matrix are functions of the time. Consequently, the transformations of velocities and accelerations are

$$
\begin{aligned}
& \delta \dot{\underline{q}}=\Phi \underline{\underline{\eta}}+\dot{\Phi} \underline{\underline{\eta}}, \\
& \delta \ddot{q}=\Phi \underline{\ddot{q}}+2 \dot{\Phi} \underline{\dot{q}}+\ddot{\Phi} \underline{\underline{\eta}} .
\end{aligned}
$$

By pre-multiplying all terms in Eq. (15) with $\Phi^{T}$ and substituting Eqs. (18) and (21), the equations of motion in the $n$ principal coordinates become

$$
\hat{M} \underline{\ddot{\eta}}+\hat{C} \underline{\underline{\eta}}+\hat{K} \underline{\eta}=\underline{\hat{\sigma}},
$$

where the modal matrices $\hat{M}, \hat{C}, \hat{K}$ and the vector of modal forces $\underline{\hat{\sigma}}$ are

$$
\begin{aligned}
\hat{M} & =\Phi^{T} \bar{M}_{0} \Phi \\
\hat{C} & =\Phi^{T} C_{0} \Phi+2 \Phi^{T} \bar{M}_{0} \dot{\Phi} \\
\hat{K} & =\Phi^{T} \bar{K}_{0} \Phi+\Phi^{T} C_{0} \dot{\Phi}+\Phi^{T} \bar{M}_{0} \ddot{\Phi} \\
\underline{\hat{\sigma}} & =\Phi^{T} \underline{\sigma}_{0} .
\end{aligned}
$$

The modal mass matrix $\hat{M}$ is diagonal. As the (possibly asymmetric) stiffness matrix $\bar{K}_{0}$ is used in Eq. (23), the term $\Phi^{T} \bar{K}_{0} \Phi$ in the expression for the modal stiffness matrix $\hat{K}$ is in general not diagonal. That will give rise to coupling between the differential equations for the natural modes in Eq. (22). Further coupling arises e.g. from the time derivatives of the modal matrix $\Phi$ and from terms with $C_{0}$. In many cases these coupling terms are relatively small. E.g. a significant contribution from $C_{0}$ is expected in high-speed rotational machinery, but not for manipulators.
In the Mode-Displacement Method (MDM) a reduced set of only $\hat{n}<n$ equations of Eq. (22) is considered. Unfortunately, in many practical cases this MDM solution is not accurate, unless many modes are taken into account. Improved convergence properties are found for the Mode-Acceleration Method (MAM). This MAM solution is obtained by computing $\hat{n}$ (low-frequency) modes dynamically as in the MDM solution. In addition, the pseudo-static response $\underline{\eta}_{s}$ of the remaining $n-\hat{n}$ (high-frequency) principal coordinates is taken into account. That is the solution of Eq. (22) ignoring the time derivatives, so

$$
\hat{K} \underline{\eta}_{s}=\underline{\hat{\sigma}} .
$$

The classification "pseudo-static response" may be misleading as $\underline{\eta}_{\mathrm{s}}$ does not represent a true steady-state configuration of the mechanism. It includes by the definition of matrix $\bar{K}_{0}$ in Eq. (17) the dynamic stiffening term $N_{0}$, which depends on (nominal) velocities and accelerations. Note also that the controller stiffness $K_{p}$ is included in $\bar{K}_{0}$ and hence in $\hat{K}$. It will usually guarantee that $\hat{K}$ is not singular and that Eq. (24) has a unique solution $\eta_{s}$.

The solution method outlined in this section will be referred to as the "Adaptive Modal Integration" (AMI) to emphasis that the change in the modal matrix $\Phi$ is taken into account in the equations of motion in the principal coordinates, see Eqs. (22) and (23).

\section{IMPLEMENTATION}

To solve the linearized equations (15) or (22), a so-called LTV block has been developed for use in a SIMULINK model [11]. Within the SIMULINK framework this LTV block represents a linear time-varying state space system

$$
\left\{\begin{array}{l}
\underline{\dot{x}}_{\mathrm{ss}}=A_{\mathrm{ss}} \underline{x}_{\mathrm{ss}}+B_{\mathrm{ss}} \underline{u}_{\mathrm{ss}} \\
\underline{y}_{\mathrm{ss}}=C_{\mathrm{ss}} \underline{x}_{\mathrm{ss}}
\end{array}\right.
$$

where $\underline{u}_{\mathrm{ss}}, \underline{y}_{\mathrm{ss}}$ and $\underline{x}_{\mathrm{ss}}$ are the input, output and state vectors, respectively, and $A_{\mathrm{ss}}, B_{\mathrm{ss}}$ and $C_{\mathrm{ss}}$ are the time-varying state space matrices.

For the equations of motion for the perturbation method, Eq. (15), the state and input vectors are defined as

$$
\underline{x}_{\mathrm{ss}}=\left[\begin{array}{c}
\delta \underline{q} \\
\delta \dot{q}
\end{array}\right], \quad \underline{u}_{\mathrm{ss}}=\left[\begin{array}{l}
\delta \underline{u}_{\mathrm{d}} \\
\underline{\sigma}_{0}^{\varepsilon m}
\end{array}\right] .
$$

The state space matrices $A_{\mathrm{ss}}$ and $B_{\mathrm{ss}}$ are then

$$
\begin{aligned}
A_{\mathrm{ss}} & =\left[\begin{array}{cc}
0 & I \\
-\bar{M}_{0}^{-1} \bar{K}_{0} & -\bar{M}_{0}^{-1} C_{0}
\end{array}\right], \\
B_{\mathrm{ss}} & =\left[\begin{array}{c}
0 \\
\bar{M}_{0}^{-1}
\end{array}\right] .
\end{aligned}
$$


The matrix $C_{\mathrm{ss}}$ depends on the definition of the output vector $y_{\mathrm{ss}}$. For the simulations in this paper $\underline{y}_{\mathrm{ss}}$ consists only of the degrees of freedom present in $\underline{x}_{s s}$ and (manipulator tip) coordinates that are computed from $\underline{x}_{\text {ss }}$ using Eq. (10).

For the AMI solution the equations of motions in principal coordinates, Eq. (22), are considered. Then, the state vector consists of the $\hat{n}$ principal coordinates $\eta_{i}$ that are computed dynamically and their derivatives $\dot{\eta}_{i}$. The input vector is defined as before in Eq. (26). The state space matrices $A_{\mathrm{ss}}$ and $B_{\mathrm{ss}}$ are now assembled using the modal matrices of Eq. (23). The dimensions of these matrices are reduced to agree with the length of the state vector, which is twice the number $\hat{n}$ of dynamically computed principal coordinates. The dimension of the matrix $C_{\mathrm{ss}}$ in the output equation is not reduced as the pseudo-static response of the remaining $n-\hat{n}$ principal coordinates must not be neglected. Consequently, there is a direct relation between input $\underline{u}_{\mathrm{ss}}$ and output $\underline{y}_{\mathrm{ss}}$. This relation is not visible in the state space equations (25), but arises as the pseudo-static solution $\eta_{\mathrm{s}}$ depends on the input signal $\delta \underline{u}_{\mathrm{d}}$, see Eqs. (16), (23), (24) and (26). Obviously, the computation of the AMI solution involves a so-called direct feedthrough [11], of which the consequences will be discussed later.

Before the SIMULINK simulation can be carried out, all time-varying coefficients in the state space representation (25) have to be determined. For that purpose the perturbation method has been added to our implementation of the finite element program SPACAR $[2,3]$. During a preprocessing run of this program the nominal motion of a rigidified manipulator is determined. The total simulation time $T$ is divided into $N$ intervals. The state space matrices and the vectors $\underline{\sigma}_{0}^{\varepsilon m}, \underline{u}_{0}$ and $\underline{y}_{0}$ are computed at the discrete time steps $t=t_{i}(i=0,1,2, \ldots, N)$. During the simulation runs the LTV block reads these data from files. Between two time steps the coefficients of the state space matrices are interpolated linearly. Cubic interpolation is used for the vectors $\underline{\sigma}_{0}^{\varepsilon m}, \underline{u}_{0}$ and $\underline{y}_{0}$. A separate tool for the modal analysis in the AMI method has been developed. The time derivatives $\dot{\Phi}$ and $\ddot{\Phi}$ of the modal matrix are computed by differentiating all coefficients in $\Phi$ numerically.

\section{SIMULATION EXAMPLE System properties}

To investigate the applicability of the perturbation method the controlled motion of a spatial two link flexible manipulator in a gravitational field is studied. Figure 1 illustrates the finite element representation of this manipulator. The revolute joints of the manipulator are modeled by the hinge elements (1), (2) and (3). The rotational axes of the hinge elements (2) and (3) are parallel, thus providing the in-plane motion of the manipulator. The vertical axis of hinge (1) coincides with the $z$ axis. The hinges are driven by internal actuators, which are modeled as pure torque sources without dynamics. The two links of the

\begin{tabular}{lrr}
\hline Beam number & 4 & 5 \\
\hline Length $l(\mathrm{~m})$ & 0.7 & 0.7 \\
Bending stiffness $E I / l^{3}(\mathrm{~N} / \mathrm{m})$ & 48300 & 24460 \\
Torsion stiffness $G I_{p} / l^{3}(\mathrm{~N} / \mathrm{m})$ & 41580 & $\infty$ \\
Mass per unit length $m(\mathrm{~kg} / \mathrm{m})$ & 4 & 2 \\
\hline
\end{tabular}

Table 1. Kinematic and dynamic link parameters.

manipulator, modeled by beam elements (4) and (5), are respectively referred to as upper arm and forearm. Both arms have uniform cross sections and are assumed to be flexible. The longitudinal deformations in both arms and the torsional deformation in the forearm are suppressed. Table 1 lists the relevant kinematic and dynamic properties of the links. The masses of the bearing assembly at the elbow hinge (3) and of the end effector are modeled by point masses of $10 \mathrm{~kg}$ and $30 \mathrm{~kg}$, respectively. The gravity loads, including the loads due to the distributed mass of the links, are taken into account by applying the corresponding external forces in the negative $z$ direction. The total model has nine flexible degrees of freedom in addition to the actuator joint coordinates $e_{1}, e_{2}$ and $e_{3}$ representing the relative rotations of the actuators.

\section{Controlled trajectory motion}

The manipulation task implies transferring the manipulator tip along a straight line with a smooth velocity profile, see Fig. 2(a) and (b). The acceleration and deceleration in the velocity profile is composed of squared sines. The torques are governed by a control algorithm consisting of an open-loop and a closed-loop component. Solving the inverse kinematic and dynamic problem of a rigidified model of the manipulator yields the necessary nominal actuator moments $\underline{u}_{0}$ of Fig. 2(c) which are applied as open-loop feedforward torques. In addition a MIMO PID feedback controller is used in which the position sensors are collocated with the actuators. The control law is given by

$$
\delta \underline{u}=-\bar{M}_{0}^{e e} H(s) \delta \underline{e}^{m},
$$

where the coupling between the actuators is represented by the time dependent mass matrix $\bar{M}_{0}^{e e}$ and $H(s)$ has only three diagonal components. Each component is a SISO PID controller

$$
H_{i}(s)=k_{p, i} \frac{\tau_{I, i} s+1}{\tau_{I, i} s} \frac{\tau_{D, i} s+1}{\alpha_{i} \tau_{D, i} s+1} \quad(i=1,2,3),
$$

where $k_{p, i}$ is the proportional gain and $\alpha_{i}, \tau_{D, i}$ and $\tau_{I, i}$ determine the zero and pole positions of the controller. For each controller 

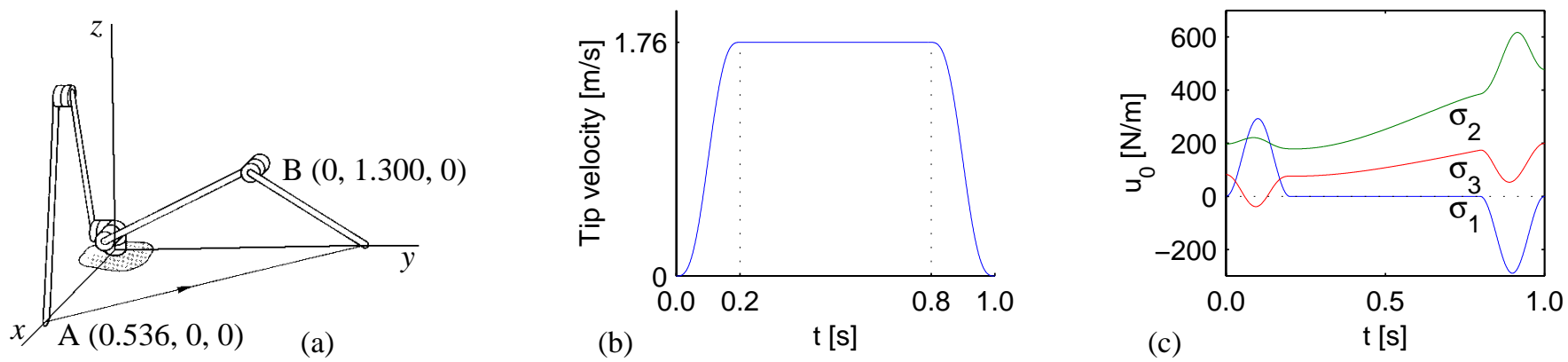

Figure 2. (a) Motion trajectory (b) velocity profile of the manipulator tip and (c) nominal torques $\underline{u}_{0}$ for the three actuators.
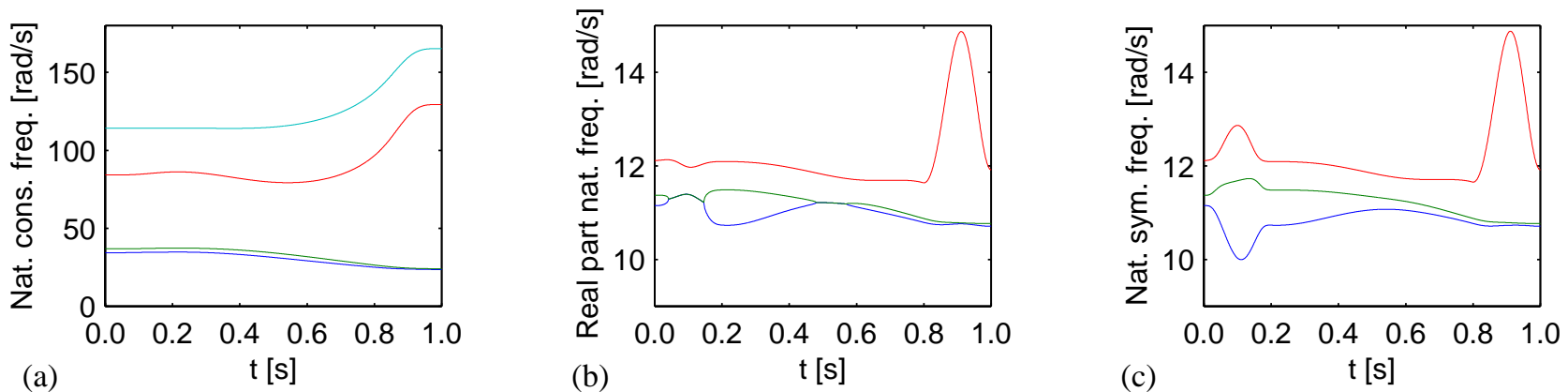

Figure 3. (a) Four lowest natural frequencies for a constraint manipulator, $\omega_{c, i}$ in Eq. (31). (b) Three lowest closed-loop natural frequencies during the controlled trajectory motion of the manipulator (only the real parts are shown). (c) As in (b) but with a symmetric $\bar{K}_{0}, \omega_{i}$ in Eq. (19).

these parameters are taken

$$
\begin{aligned}
k_{p, i} & =\omega_{b, i}^{2}, \\
\tau_{I, i} & =6 / \omega_{b, i}, \\
\tau_{D, i} & =1.5 / \omega_{b, i}, \\
\alpha_{i} & =0.1,
\end{aligned}
$$

in which $\omega_{b, i}$ are the desired servo loop frequencies. In principal each loop can be tuned separately, where $\omega_{b, i}$ should be chosen sufficiently low with respect to the natural frequencies of the system to avoid instabilities.

The inertia properties depend on the configuration, so the mass matrix $\bar{M}_{0}^{e e}$ in Eq. (28) changes during the simulation. For the specified initial and final configuration some of the terms differ by a factor of 6 . Hence the mass matrix $\bar{M}_{0}^{e e}$ has to be updated during the motion.

Analogous to the approach of Book et al. [12] the servo loop frequencies $\omega_{b, i}$ are chosen with respect to the lowest constraint natural frequencies $\omega_{c, i}$ of the system. In the constraint system all actuator joint coordinates $\underline{e}^{m}$ are prescribed and thus $\delta \underline{e}^{m}=0$. The associated frequency equation is then given by

$$
\operatorname{det}\left(-\omega_{c, i}^{2} \bar{M}_{0}^{\varepsilon \varepsilon}+\bar{K}_{0}^{\varepsilon \varepsilon}\right)=0 .
$$

For the considered motion the lowest frequency changes only gradually from $34 \mathrm{rad} / \mathrm{s}$ to $24 \mathrm{rad} / \mathrm{s}$, see Fig. 3(a). With fixed setting $\omega_{b}=12 \mathrm{rad} / \mathrm{s}$ for all $\omega_{b, i}$ a stable controller is expected. The proportional part of the control law is then

$$
K_{p}=\omega_{b}^{2} M_{0}^{e e} .
$$

Using this value the natural frequencies of Eq. (19) have been calculated and the lowest three closed-loop natural frequencies are shown in Fig. 3(c). The increase of the third natural frequency near $t=0.9 \mathrm{~s}$ is caused by the dynamic stiffening. It should be noted that these natural frequencies are computed with the symmetric stiffness matrix $\bar{K}_{0}^{S}$. In case the true stiffness matrix $\bar{K}_{0}$ is used, the natural frequencies of Fig. 3(b) are found. Near $t=0.1 \mathrm{~s}$ and $0.6 \mathrm{~s}$ the two lowest natural frequencies have imaginary parts and they are each others complex conjugates. In the graph only the real parts are shown and they coincide.

\section{Simulation results}

The simulations have been carried out using SIMULINK's ode 45 variable time step Runge-Kutta solver with the relative tolerance set to $10^{-4}$ [11]. Results from three methods are compared: the non-linear finite element method (Eq. (7)), the perturbation method (Eq. (15)) with the number of linearization points $N=1500$ and the AMI method (Eqs. (22) and (24)) with $\hat{n}=4$ (the number of modes in Eq. (22)). In a previous paper [4] we 

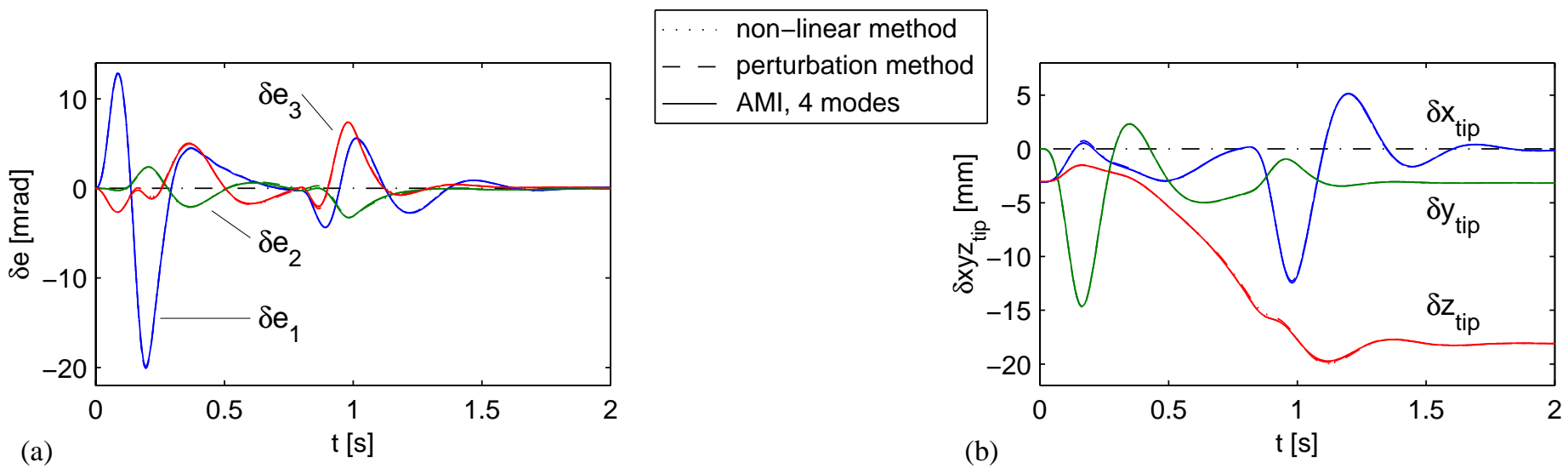

Figure 4. Deviation from the nominal trajectory according to three simulation methods: (a) actuator rotations and (b) tip coordinates.

showed that using less linearization points leads to larger differences between the non-linear simulation and the perturbation method for this manipulator motion with a PD controller. Increasing $N$ further did not improve the accuracy.

Figure 4 shows the deviation from the nominal trajectory for the actuator angles and the manipulator tip position according to these three methods. In all simulations the manipulator is at rest at $t=0 \mathrm{~s}$. The integral action of the PID controllers results in zero errors for the actuator angles. The initial deformations of the links are computed from the steady solution which is found by splitting the steady part of Eq. (15) into

$$
\begin{aligned}
& \bar{K}_{0}^{e e} \delta \underline{e}^{m}+\bar{K}_{0}^{e \varepsilon} \underline{\varepsilon}^{m}=\delta \underline{u}_{\mathrm{d}} \\
& \bar{K}_{0}^{\varepsilon e} \delta \underline{e}^{m}+\bar{K}_{0}^{\varepsilon \varepsilon} \underline{\varepsilon}^{m}=\underline{\sigma}_{0}^{\varepsilon m}
\end{aligned}
$$

As $\delta \underline{e}^{m}=0$, the link deformations $\underline{\varepsilon}^{m}$ can be solved from the second set of equations. The initial integral controller action $\delta \underline{u}_{\mathrm{d}}$ follows then immediately from the first set of equations. The deviations from the nominal position of the link are in the $x z$ plane, which is clearly expressed by the non-zero $\delta x_{\text {tip }}$ and $\delta z_{\text {tip }}$ at $t=0 \mathrm{~s}$ in Fig. 4(b). For $0 \mathrm{~s}<t<1 \mathrm{~s}$ the manipulator moves along the trajectory. The effects of acceleration and deceleration can be seen in Fig. 4. Clearly, all simulations show that the proposed controller is stable and the manipulator is practically at rest for $t>1.7 \mathrm{~s}$. The deviations from the nominal position are now in the $y z$-plane, which results in non-zero $\delta y_{\text {tip }}$ and $\delta z_{\text {tip }}$. Obviously, the deviation in the $z$ direction is larger than at $t=0 \mathrm{~s}$ as the distance of the tip of the manipulator to the origin is larger.

Some differences are found between the simulations in Fig. 4. The $z$ position for $t \approx 0.85 \mathrm{~s}$ is slightly lower according to the perturbation method than the non-linear result. This error of approximately $0.26 \mathrm{~mm}$ is hardly visible on the scale of the graph. It illustrates the accuracy that can be obtained with the linearized model. Subsequent modal reduction to 4 modes does not affect the accuracy as the results from the AMI method can not be distinguished from the perturbation method in Fig. 4. The maximum deviation from the perturbation method is found for $\delta x_{\text {tip }}$ near $t=1.04 \mathrm{~s}$ and is about $0.15 \mathrm{~mm}$.

The error introduced by the modal reduction is illustrated in more detail in Fig. 5 where for each tip coordinate the maximum error during the complete trajectory motion is plotted as a function of the number of modes. Obviously, by taking into account only 3 modes the error is already comparable to the error that was introduced by the linearization. Simulating with more modes only leads to the inclusion of modes with higher natural frequencies which slow down the simulations without improving the accuracy. This is demonstrated clearly in Fig. 6 which shows the required CPU time and the number of time steps for the simulations. A simulation using the AMI method with 3 or 4 modes is efficient as the $5^{\text {th }}$ mode apparently leads to a significant increase in both the required CPU time and the number of time steps, but does not improve the accuracy (Fig. 5). Note that an AMI simulation with 11 modes needs more CPU time than the AMI and perturbation method simulations with all modes. The reason for this is the direct feedthrough from the input $\delta \underline{u}_{\mathrm{d}}$ to the output $\delta \underline{e}^{m}$ due to the pseudo-static solution (24) of the $n-\hat{n}$ high-frequency modes. The applied PID controllers of Eq. (29) also have a direct coupling between input and output, which remains when the proportional part of Eq. (32) has been removed. Then an algebraic loop exists and SIMULINK has to call an iterative loop solving routine at each time step which slows down the simulations [11].

\section{CONCLUSIONS}

The presented perturbation method permits an efficient numerical simulation of the controlled trajectory motion of a flexible manipulator as well as a straightforward vibration control formulation. A further reduction of the simulation time was obtained by applying a modal reduction technique, which we refer to as the Adaptive Modal Integration (AMI) method. For the spatial flexible two link manipulator, results of both the perturbation 


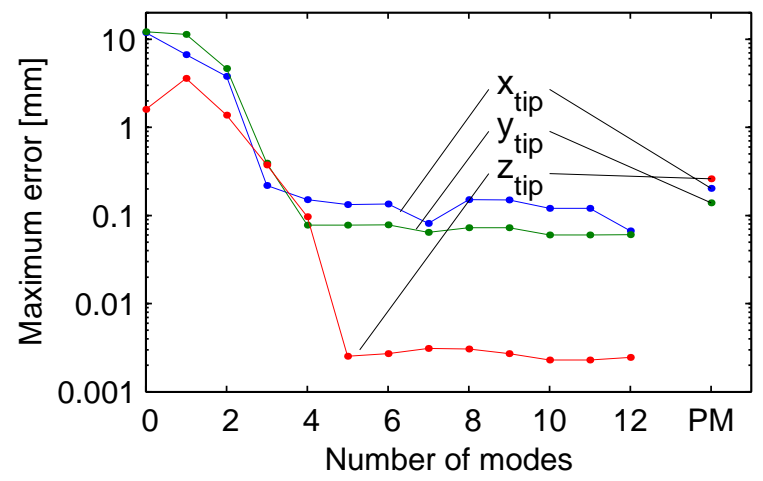

Figure 5. Maximum errors along the whole trajectory in the coordinates of the tip position as functions of the number of modes. The error is computed by comparing the AMI method with the perturbation method. For comparison the dots indicated with "PM" show the maximum difference between the perturbation method and the non-linear simulation.

method and the AMI method agree well with the results obtained from a full non-linear analysis. In the AMI method only three (modified rigid link) of four degrees of freedom are needed to reach a satisfying accuracy. Crucial elements in the AMI method are the availability of accurate linearized equations and a careful modal analysis in which the time-varying nature of the mode shape functions and the proportional feedback gains are taken into account.

\section{ACKNOWLEDGMENT}

The authors acknowledge the work carried out by R.R. Waiboer and $\mathrm{H}$. Super on the implementation and simulations of the perturbation method with modal analysis.

\section{REFERENCES}

[1] Jonker, J. B., 1990, "A finite element dynamic analysis of flexible manipulators", International Journal of Robotics Research, 9(4), pp. 59-74.

[2] Jonker, J.B., and Meijaard, J.P., 1990, "SPACAR-computer program for dynamic analysis of flexible spatial mechanisms and manipulators", Multibody systems handbook, W. Schiehlen, ed., Springer-Verlag, Berlin, pp. 123-143.

[3] Jonker, J.B. and Aarts, R.G.K.M., 1998, "Modelling of flexible mechanisms and manipulators for control purposes", Proceedings of the Fourth International Conference on Motion and Vibration Control, MoViC, Institute of Robotics, ETHZurich, Vol. 1, pp. 291-297.

[4] Jonker, J.B. and Aarts, R.G.K.M., 2001, "A perturbation method for dynamic analysis and simulation of flexible manipulators", Multibody Systems Dynamics, accepted for publication.

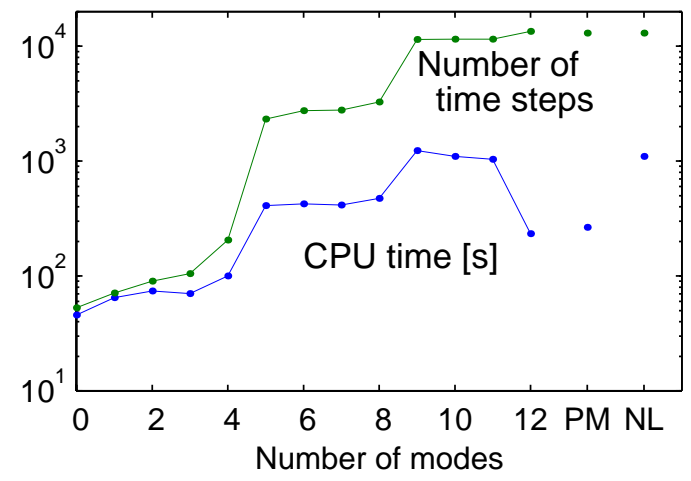

Figure 6. Required CPU time and number of time steps during the simulation with the AMI method as functions of the number of modes. For comparison the perturbation method and the non-linear simulation are included and are indicated with "PM" and "NL", respectively.

[5] Meijaard, J.P., 1996, "Validation of flexible beam elements in dynamics programs", Nonlinear Dynamics, 9, pp. 21-36.

[6] Jonker, J.B., 1989, "A finite element dynamic analysis of spatial mechanisms with flexible links", Computer Methods in applied Mechanics and Engineering, 76, pp. 17-40.

[7] Meijaard, J. P., 1991, "Direct determination of periodic solutions of the dynamical equations of flexible mechanisms and manipulators", International Journal of Numerical Methods in Engineering, 32, pp. 1691-1710.

[8] Jonker, J.B., 1991, "Linearization of dynamic equations of flexible mechanisms - a finite element approach", International Journal of Numerical Methods in Engineering, 31, pp. 13751392.

[9] Craig, R.R., Jr., 1981, Structural Dynamics: An Introduction to Computer Methods, John Wiley and Sons, New York.

[10] Meirovitch, L., 1980, Computational methods in structural dynamics, Sijthoff \& Noordhoff, Alphen aan den Rijn, The Netherlands.

[11] Simulink, 1999, Dynamic System Simulation for MATLAB, Using SIMULINK version 3, The MathWorks Inc., Natick (MA).

[12] Book, W.J., O. Maizza-Neto and D.E. Withney, 1975, "Feedback control of two beam, two joint systems with distributed flexibility", Journal of Dynamic Systems, Measurement and Control, 97, pp. 424-431. 